little water, full of ordinary air, and provided with means of ex panding the air in the flask, and either returning the air to the flask, or admitting filtered air. Go on repeating the process of expanding and cloud-making in the flask. After this has been done a number of times, the nuclei become fewer and fewer, and at last only a very few are left in the air. Every one must have noticed when making this experiment that the cloud particles are very small on the first expansion, and that they fall very slowly, almost imperceptibly, but that at the end of the experiment, when the last dust particles become nuclei, the water particles are large and fall rapidly like rain drops. At the beginning of the experiment, with plenty of dust in the air, there is almost no supersaturation, the nuclei being so close the tension is relieved as soon as it is formed. When, however, only a few particles are present, there are large spaces between the nuclei where supersaturation can take place, and it is by falling through this supersaturated air that the drops, when few in number, are able to grow so quickly and become so large. It therefore seems probable that something of the same kind will happen if ions were to become nuclei in supersaturated air. Whenever an ion becomes active it will rapidly grow to the dimensions of a rain-drop in the same manner and for the same reason that the dust-nucleused drops do in supersaturated air. These little drops evidently have a way of parting with the heat of condensation at a very much quicker rate than Mr. Wilson is disposed to admit.

It is this capacity for rapid growth in supersaturated air that makes it so improbable that ions can ever give rise to a cloudy form of condensation. To form a cloud a large number of them would require to become active at the same moment. But this is evidently not possible in a rising column of air. The ions which rise on the top of the ascending column will become active first, and by falling through the lower supersaturated air will grow with great rapidity and give rise to a rainy, but cloudless form of condensation.

There are some points connected with ions about which I think the readers of NATURE would be glad to have some information, and which I think Mr. Wilson, with the aid of the apparatus at his disposal, could give us. For instance, cne would like to know (I) how long ions remain in air in an inclosed vessel, when both + and - ions are present ; $(2)$ when only + or - ions are in the air ; (3) whether the presence of dust has any effect on the duration of their life. For practical purposes one would also like to know further (I) how many ions are generally in the air near the ground ; $(2)$ what amount of electricity they carry with them.

Finally, one would like to know how many ions will pass up through a cloud and escape at the top; as one would almost expect, these ions, with their electric charges, will be more likely to be cleared out of the a ir by rain than the dust particles, and whether both kinds are equally liable to be washed out by rain. If not, the inequality may help to explain some important electrical phenomena.

Ardenlea, Falkirk, June 27.

The Melting Points of Rock-forming Minerals.

IN connection with the abstracts of papers read before the Royal Dublin Society by Dr. J. Joly, F.R.S., and myself, given in NATURE for July I 2 (p. 262), I might perhaps be permitted to draw attention to a few points. The same subject has been recently dealt with by $\mathrm{Mr}$. C. E. Stroneyer (Mem. Manchester Lit. and Phil. Soc., vol. xliv. Part iii. No. 7, 1900) and by Prof. Sollas, F.R.S. (Geol. Mas., July 1900).

In the first place it may be noted that the "melting point" of a substance under a definite pressure has a perfectly definite meaning. The "softening point," on the other hand, obviously depends on the magnitude of the distorting force with which the softness is tested, as weil as on the other conditions of experi. ment.

It is an established fact that the melting points of a very large number of substances vary with the pressure. Bunsen, as far back as 1850 , perceived the geological application of this phenomenon. In discussing the crystallisation of plutonic rocks, it is the melting points of the minerals under enormous pressures which really concern us. These pressures are probably sufficient to alter the melting points through several hundred degrees. There are then two ways open for us to ascertain these melting points. Firstly, we might determine them by direct experiment at the necessary large pressures; or, secondly, we might measure the melting points at ordinary atmospheric pressure and determine the rate of increase (or decrease) of melting point with increase of pressure $(d \theta / d p)$. Considering the gigantic pressures with which we have to deal, it seems decidedly easier to adopt the second method. The agreement between the results obtained from the application of the thermodynamic formula

$$
\frac{d \theta}{d p}=\frac{\theta\left(\nu_{1}-\tau_{s}\right)}{\mathrm{L}}
$$

(where $\theta=$ absolute melting temperature; $\left(v_{l}-v_{s}\right)=$ the change of volume at the instant of melting; $L=$ the latent heat in mechanical units) with the results of experiments (e.g. M. A. Battelli, Journal de Phys., t. viii. p. 90, I887), seems to justify the application of that formula to the case of the minerals in question, in the absence of direct experiment. It is true that the formula was deduced for a reversible system, and that no natural process is reversible. But a similar objection would hold against the application of any theoretical formula to the conditions obtainable in experimental work. In the present case it is only claimed for the formula that it will afford an approximate estimate of the melting points of minerals under large pressures; and after all, even direct measurement of such high temperatures as are involved is always attended with un. certainty. In order to apply this formula we require $\theta,\left(v_{l}-v_{s}\right)$, and L. The melting points of the most important minerals at atmospheric pressure have been determined by Dr. Joly and Mr. R. Cusack (Proc. Roy. Irish Acad., Ser. 3. vol. ii. p. 38 ; vol. iv. p. 399). A large part of the volume change on melting is, I submit, afforded us by the difference in density between the crystalline mineral and its fused glass. Now it is characteristic of amorphous substances to pass gradually and continuously from solid to liquid ( $c f$. Preston, "Theory of Heat," pp. 270 and 286); and so it is highly probable that such a mineral glass will pass without sudden volume change into the liquid state, and it has, in fact, passed gradually in the inverse direction. It is not contended that any given mineral ever existed as a glass in the molten magma of an igneous rock, but only that it existed as a liquid.

In my paper, above referred to, I have shown how the "fusibility" of a mineral must be connected with its latent heat, and hence by a comparison of relative fusibility and melting temperature we may often deduce the relative latent heats of two minerals. Thus, for example, the "fusibility" of labradorite is 3 on von Kobell's scale, and its melting point is $1229^{\circ} \mathrm{C}$., whereas orthoclase has a inelting point of only $1175^{\circ} \mathrm{C}$., but is much less "fusible," viz. 5 on von Kobell's scale. Hence I infer that the latent heat of orthoclase is decidedly greater than that of labradorite. Similarly, the latent heat of augite is less than that of orthoclase. But the volume-change on melting of augite is greater than that of orthoclase. Therefore $d \theta / d p$ is greater for augite than for orthoclase. It is thus possible to arrive at the order of melting points of minerals under the pressure of rock formation. If, after ascertaining this order, it is still found to be inconsistent with the order of crystallisation, as shown by microsopical examination, it may be necessary to examine the more complicated influences of solution, \&c., on the crystallising points of the minerals.

In conclusion, I may point out that it must be a matter of extreme importance in measuring the melting temperature of quartz to make sure that the specimen used is pure, and in particular free from the alkalis. Messrs. Shenstone and Lacell (NATURE, May 3, I900, p. 20) have found that rock crystal very often contains sodium and lithium, traces of which might be expected to lower the melting point. Further, it has long been known that quartz, with a density of $2 \cdot 66$, passes into the variety of silica with density $2 \cdot 3$ at a temperature below its melting point (cf. Fremy, Enc. Chim. 6, p. I42). And similar transformations are common among metals. Is it not possible then that the phenomena observed by Dr. Joly may have nothing to do with the fusion point of quartz, but are simply cases of molecular transformation at a temperature below the melting point ?

Royal College of Science, Dublin.

\section{Observation of the Circular Components in the} "Faraday Effect."

AFTER repeated attempts to determine the nature of the "Faraday effect," I have succeeded in observing that ordinary light, when passing from a surface into a medium in such a way

$$
\text { NO. } 1607, \text { VOL. } 62]
$$

